\title{
Transport properties of two-dimensional electron systems on silicon (111) surfaces
}

\author{
E. H. Hwang and S. Das Sarma \\ Condensed Matter Theory Center, Department of Physics, University of Maryland, College Park, Maryland 20742-4111
}

(July 27, 2021)

\begin{abstract}
We theoretically study transport properties of a two-dimensional electron system on a hydrogenpassivated $\mathrm{Si}(111)$ surface in the field-effect-transistor (FET) configuration. We calculate the density and temperature dependent mobility and resistivity for the recently fabricated Si(111)-vacuum FET by using a semiclassical Boltzmann theory including screened charged impurity scattering. We find reasonable agreement with the corresponding experimental transport properties, indicating that the screened disorder potential from random charged impurities is the main scattering mechanism. We also find that the theoretical results with the valley degeneracy $g_{v}=2$ give much better agreement with experiment than the $g_{v}=6$ situation indicating that the usual bulk six-valley degeneracy of $\mathrm{Si}$ is lifted in this system.
\end{abstract}

PACS Number : 73.40.Qv, 72.10.-d, 73.40.-c

Recently a high mobility two dimensional electron system (2DES) on $\mathrm{Si}(111)$ surface has been fabricated [1,2]. The fabricated Si-vacuum field-effect transistor (FET), in which an electric field is applied through an encapsulated vacuum cavity instead of the usual $\mathrm{SiO}_{2}$ as in a $\mathrm{Si}$ MOSFET, allows the 2DES to be gated on a hydrogenpassivated $\mathrm{Si}(111)$ surface. The strong scattering potential associated with the disordered $\mathrm{Si}_{-} \mathrm{SiO}_{2}$ interface in the usual Si MOSFET geometry [3] being absent at the H-passivated $\mathrm{Si}(111)$-vacuum interface, the carrier mobility is expected to be higher in this new 2D system compared with the extensively-studied [3] $\mathrm{Si}(111)-\mathrm{SiO}_{2} 2 \mathrm{D}$ MOSFET system. In the usual $\mathrm{Si}-\mathrm{SiO}_{2}$ MOSFETs the carrier mobility is limited by charged impurity scattering at low carrier densities, but at higher carrier densities, when the $2 \mathrm{DES}$ is pushed very close to the $\mathrm{Si}-\mathrm{SiO}_{2}$ interface by the steep self-consistent confining potential arising from the carriers themselves, surface roughness and defect scattering associated with the $\mathrm{Si}_{-} \mathrm{SiO}_{2}$ interface becomes dominant, reducing carrier mobility with increasing density. The 2D mobility in $\mathrm{Si}_{-} \mathrm{SiO}_{2}$ MOSFETs therefore has a peak value at some intermediate carrier density $\left(\sim 10^{12} \mathrm{~cm}^{-2}\right)$ with the mobility limited by charged impurity scattering (interface roughness scattering) below (above) the characteristic density where the 2D mobility attains its peak value. Potential advantages of the newly created $[1,2]$ Si-vacuum FET structure are that the absence of interface roughness/dangling bond scattering leads to (1) very high $2 \mathrm{D}$ mobilities not achievable in Si$\mathrm{SiO}_{2}$ MOSFET structures; and (2) the mobility should, in principle, be a monotonically increasing function of 2D carrier density, as, for example, in 2D undoped GaAs HIGFET structures [4]. In the Si-vacuum FET fabricated by Eng et al. [1,2] the $\mathrm{H}-\mathrm{Si}(111)$ surface reduces surface scattering, thereby increasing electron mobility. The measured [1] peak electron mobility on $\mathrm{H}-\mathrm{Si}(111)$ vacuum FET reaches $\sim 8000 \mathrm{~cm}^{2} / \mathrm{Vs}$ at $4.2 \mathrm{~K}$, which is the highest electron mobility recorded on a $\mathrm{Si}(111)$ surface compared to the previous peak electron mobility measurements in $\mathrm{Si}(111)$ MOSFETs $\left(\sim 2500 \mathrm{~cm}^{2} / \mathrm{Vs}\right)$ $[5-7]$. With this enhanced electron mobility, the Sivacuum FET has potential development of atomic-scale devices, where electrons are coupled to specific molecules or single atoms positioned on the H-passivated Si surface. Indeed, with further improvement in the surface passivation techniques developed in refs. [1,2], the experimental mobilities should go up much higher than the currently published [1] record of $\sim 8000 \mathrm{~cm}^{2} / \mathrm{Vs}$.

In contrast to the well-studied electron inversion layers in $\mathrm{Si}(100)$ MOSFETs [3], the detailed investigation of electronic transport properties of $2 \mathrm{DES}$ on $\mathrm{Si}(111)$ surface has not been reported in the literature. Since the high mobility FET on $\mathrm{Si}(111)$ surface is now available, a detailed study of electronic transport properties is necessary. The purpose of this paper is to calculate the density and temperature dependent electron mobility of the 2DES on $\mathrm{Si}(111)$ surface. We also calculate the $2 \mathrm{D}$ temperature-dependent resistivity in connection with the subject of $2 \mathrm{D}$ metal-insulator transition and the 2D metallic phase which has been studied for most other high mobility 2D systems [8]. The measured temperature dependent resistivity in Si-vacuum FET shows strong metallic behavior [2] (i.e. the resistivity increases with temperature) with no sign of insulating behavior down to a density $n=3.7 \times 10^{11} \mathrm{~cm}^{-2}$ (which is the lowest achieved experimental density so far). The density dependence of mobility in the metallic regime of the Sivacuum FET may give valuable information about the scattering mechanisms operational in limiting 2D carrier transport, since different scattering sources give rise to different density dependence in the resistivity. Thus, a detailed study of the density and temperature dependence of $2 \mathrm{D}$ resistivity in high-mobility $2 \mathrm{D} \mathrm{Si}(111)$ vacuum FET could be useful not only for a better fundamental understanding of the limiting transport mechanisms in this potentially important new system, but also for providing ideas about how to further increase the $2 \mathrm{D}$ mobility leading to better devices. 
In order to calculate the low temperature $2 \mathrm{D}$ electron mobility we have carried out a microscopic transport calculation using the Boltzmann transport theory [8]. We calculate the mobility in the presence of randomly distributed Coulomb impurity charges near the surface and the bulk-acceptors in the depletion layer with the electron-impurity interaction being screened by the $2 \mathrm{D}$ electron gas in the random phase approximation (RPA). The screened Coulomb scattering is the only important scattering mechanism in our calculation. There are additional interface-scattering mechanisms unrelated to the Coulomb centers (e.g. surface roughness scattering), but such interface scattering would become more pronounced at higher densities, as the inversion layer is drawn closer to the interface. In general, the surface roughness scattering gives rise to the decrease of the mobility at high densities. The observed mobility [1] shows that the mobility increases monotonically with electron density indicating the apparent absence of interface scattering, as expected for a Si-vacuum interface. In fact, as argued above in this paper, the surface roughness scattering should be negligible in the Si-vacuum FET systems, allowing us to ignore this process in our theory. We also neglect all phonon scattering effects mainly because our theoretical estimate shows phonon scattering to be negligible for Si-vacuum FET structures in the $T<10 K$ regime of interest to us. We note that our theoretical approach has provided a reasonable description of the transport properties for several $2 \mathrm{D}$ systems [8].

In the Boltzmann theory the mobility is given by $\mu=e\langle\tau\rangle / m$ with $m$ as the carrier effective mass, and $\langle\tau\rangle$ the energy averaged finite temperature scattering time: $\langle\tau\rangle=\int d \epsilon_{k} \epsilon_{k} \tau\left(\epsilon_{k}\right)\left(-\frac{\partial f}{\partial \epsilon_{k}}\right) / \int d \epsilon_{k} \epsilon_{k}\left(-\frac{\partial f}{\partial \epsilon_{k}}\right)$, where $f\left(\epsilon_{k}\right)$ is the Fermi distribution function, $f\left(\epsilon_{k}\right)=\left\{1+\exp \left[\left(\epsilon_{k}-\right.\right.\right.$ $\left.\mu)] / k_{B} T\right\}^{-1}$ with $\mu(T, n)$ as the finite temperature chemical potential determined self-consistently. The energy dependent scattering time $\tau\left(\epsilon_{k}\right)$ for our model of randomly distributed impurity charge centers is given in the leading-order theory by

$$
\begin{aligned}
\frac{1}{\tau\left(\epsilon_{k}\right)}= & \frac{2 \pi}{\hbar} \sum_{a} \int n_{i}^{(a)}(z) d z \int \frac{d^{2} k^{\prime}}{(2 \pi)^{2}}\left|\frac{v(q, z)}{\varepsilon(q)}\right|^{2} \\
& \times(1-\cos \theta) \delta\left(\epsilon_{\mathbf{k}}-\epsilon_{\mathbf{k}^{\prime}}\right)
\end{aligned}
$$

where $n_{i}^{(a)}$ is the concentration of the $a$-th kind of charged impurity center, $q=\left|\mathbf{k}-\mathbf{k}^{\prime}\right|, \theta \equiv \theta_{\mathbf{k k}^{\prime}}$ is the scattering angle between the scattering in- and out- wave vectors $\mathbf{k}$ and $\mathbf{k}^{\prime}, \epsilon_{\mathbf{k}}=\hbar^{2} k^{2} / 2 m, v(q, z)$ is the $2 \mathrm{D}$ Coulomb interaction between an electron and an impurity at $z$, and $\varepsilon(q) \equiv \varepsilon(q ; \mu, T)$ is the 2D finite temperature static RPA dielectric (screening) function [3]. In calculating the Coulomb interaction and the RPA dielectric function in Eq. (1) we take into account subband quantization effects in the inversion layer through the lowest subband variational wavefunction [3]. From calculated mobility we get the resistivity given by $\rho=1 /$ ne $\mu$.

Throughout this paper we use the following material parameters: valley degeneracy $g_{v}=2$ (or $g_{v}=6$ as the case may be), an effective mass $m=0.35 m_{e}$ (corresponding to the $\mathrm{Si}(111)$ surface) where $m_{e}$ is the free electron mass, $\kappa_{S i}=11.7$ and $\kappa_{v a c}=1$, the dielectric constant of $\mathrm{Si}$ and vacuum, respectively. Even though the valley degeneracy $\left(g_{v}\right)$ on the $\mathrm{Si}(100)$ surface is well-known to be $g_{v}=2$, the valley degeneracy on the $\mathrm{Si}(111)$ surface has been a puzzle for a long time [3]. Since Si has six conduction-band minima located along the (100) directions, the (111) surface should have six equivalent valleys (i.e. $g_{v}=6$ ) within the simple effective mass approximation. However, magnetotransport measurements have found the valley degeneracy to be both 2 and $6[5-7,3]$, with most experiments finding the lower valley degeneracy of 2 . In our calculation we get much better agreement with the experimental data of refs. $[1,2]$ when we use the valley degeneracy $g_{v}=2$ instead of $g_{v}=6$, indicating that the valley degeneracy is reduced from 6 to 2 in the Si(111)-vacuum FET structures of ref. [1].

In Fig. 1 we show the calculated mobility as a function of electron density for different temperatures. The mobilities for Si(111)-vacuum FET are shown in Fig. 1(a) with valley degeneracy $g_{v}=2$ and Fig. $1(\mathrm{~b})$ with $g_{v}=6$. In Fig. 1(c) we show the mobility of a Si(111) MOSFET system with $g_{v}=2$. For the $\mathrm{Si}_{-} \mathrm{SiO}_{2}$ MOSFET calculations we use $\kappa_{\mathrm{SiO}_{2}}=3.9$ with the other parameters being the same as in the Si-vacuum system. In Fig. 1 the mobilities are calculated with fixed impurity density $n_{i}=7 \times 10^{11} \mathrm{~cm}^{-2}$ (the impurity centers are distributed completely at random in a $2 \mathrm{D}$ plane at $d=50 \AA$ ) and the ionized impurity density $n_{3 D}=4 \times 10^{16} \mathrm{~cm}^{-3}$ in the depletion layer of the $p$-type Si. Here $d$ indicates the distance between the impurity charge centers and the inversion layer. This is intended to represent the average distance between an electron in the inversion layer and the impurity near the Si-vacuum interface. The calculated mobilities depend on the density approximately as $\mu \sim n^{\alpha}$ for the calculated density range. We emphasize that this is not a strict power law since the exponent $\alpha$ depends weakly on the density. Over the experimental density range, we find that for $g_{v}=2$ [Fig. 1(a)] the exponent $\alpha$ in $\mu \propto n^{\alpha}$ is about 0.7 at $\mathrm{T}=0 \mathrm{~K}$ and increases as temperature increases. At $T=4 K$ we have $\alpha \approx 1.2$ which is consistent with experiment [1,2]. For $g_{v}=6$ [Fig. $\left.1(\mathrm{~b})\right]$ we have $\alpha \approx 0.5-1.5$. Note that the $\mathrm{Si}(111)$ MOSFET system [Fig. $1(\mathrm{c})$ ] has $\alpha \approx 0.3-0.6$ in the given temperature range. [The characteristic separation "d" between the charged impurities and the 2DES as well as the random impurity densities $n_{i}$ and $n_{3 D}$ are of course not precisely known for the experimental samples, but the parameters we use are quite typical, and give decent agreement with the experimental results of ref. [1] for $g_{v}=2$, i.e. Fig. 1(a).] Our mobility calculation indicates that the screened potential from random 

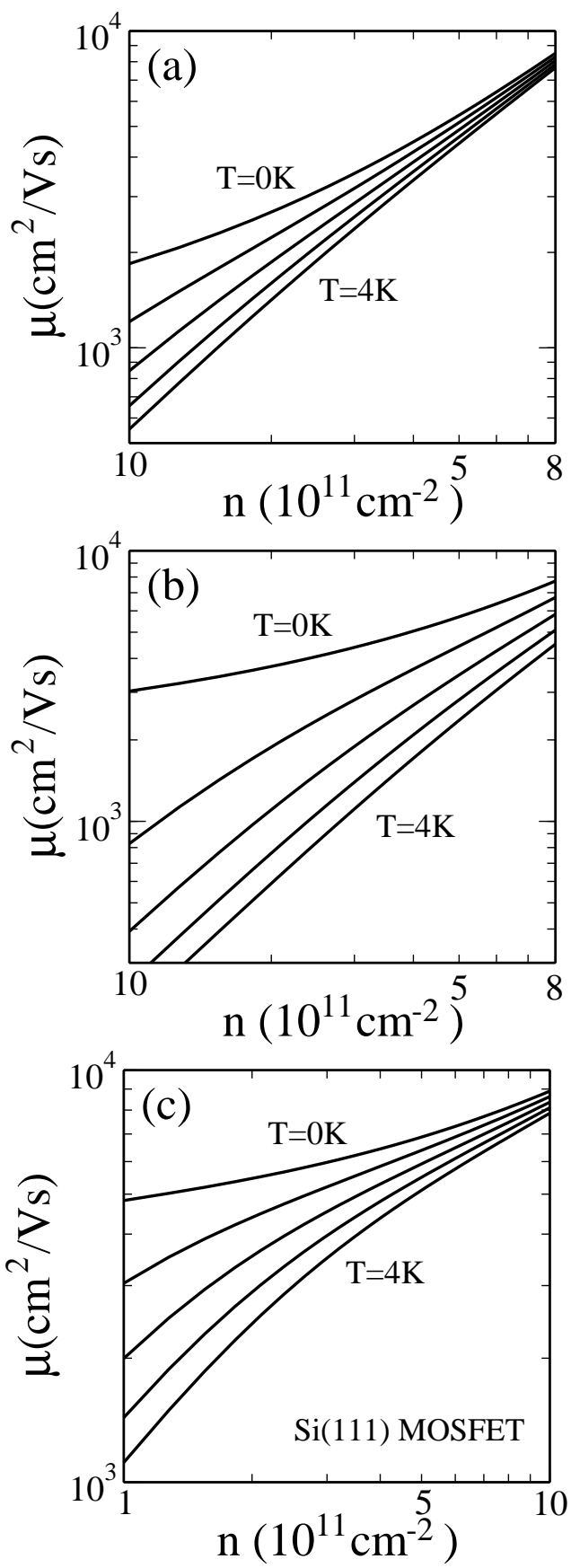

FIG. 1. Calculated electron mobility in the $\mathrm{Si}(111)$-vacuum FET system for various temperatures, $T=0,1,2,3,4 \mathrm{~K}$ (top to bottom) with valley degeneracy (a) $g_{v}=2$ and (b) $g_{v}=6$. Fig. 1(c) shows the calculated electron mobility for a $\mathrm{Si}(111)-\mathrm{SiO}_{2}$ MOSFET with $g_{v}=2$. The calculated mobilities depend on the density approximately as $\mu \sim n^{\alpha}$ with $\alpha \approx 0.7$ at $T=0$ and $\alpha \approx 1.2$ at $T=4 K$ for $g_{v}=2$ [Fig. 1(a)], and $\alpha \approx 0.5-1.5$ for $g_{v}=6$ [Fig. 1(b)]. For $\mathrm{Si}(111) \operatorname{MOSFET}[$ Fig. $1(\mathrm{c})$ ] we have $\alpha \approx 0.3-0.6$. Note that surface roughness scattering, known to be important for $\mathrm{Si}-\mathrm{SiO}_{2}$ MOSFETs at higher carrier densities, is neglected in the calculation.

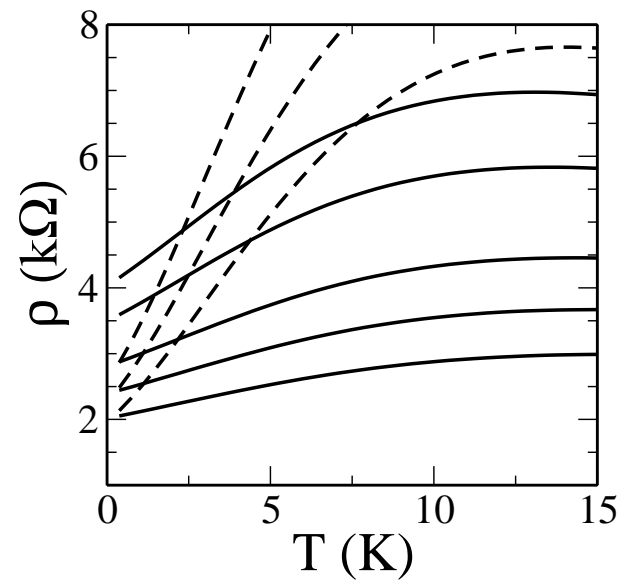

FIG. 2. Calculated resistivities for various hole densities $n=3.7,4.0,4.5,4.9,5.36 \times 10^{11} \mathrm{~cm}^{-2}$ (from top to bottom) with the same parameters of Fig. 1. The dashed lines show the calculated resistivity for density $n=4.5,4.9$, $5.36 \times 10^{11} \mathrm{~cm}^{-2}$ with the valley degeneracy $g_{v}=6$.

charged impurities is the main scattering source and the $\operatorname{Si}(111)$-vacuum FET has a valley degeneracy $g_{v}=2$. Since there are no adjustable parameters in our calculation except the impurity parameters, the agreement is quite good. The calculated mobility obviously depends on the distance $(d)$ of the charged impurity centers from the 2D layer. Generally the location of the charged impurities in the FET is not known. Thus, the choice of $d=50 \AA$ is reasonable because our calculated mobility has the same dependence on $n$ as does the measured mobility $[1,2]$.

In Fig. 2 we show the calculated temperature dependent resistivity using the same parameters as in Fig. 1. The calculated resistivity increases as the temperature increases (i.e. metallic behavior), and shows an approximately linear temperature dependence in the $T<5 \mathrm{~K}$ regime as observed in the experiment [2]. The temperature dependence of $\rho(T)$ becomes stronger with decreasing density just as the experimental observation in $\mathrm{Si}(111)$-vacuum FET [2]. This strong linear temperature dependence of $\rho(T)$ in this system arises $[8,9]$ due to the peculiar nature of the $2 \mathrm{D}$ screening function which has a cusp at wave vector $q=2 k_{F}$. Since $2 k_{F}$ scattering is the most dominant resistive scattering at low temperatures, thermal smearing of this $2 k_{F}$ cusp leads to strong temperature dependence of resistivity. Thus, temperature dependent screening of charged impurity scattering in 2D layers leads to a strongly temperature dependent effective disorder controlling $\rho(T, n)$ at low temperatures and densities. (The screening effect decreases with increasing temperature giving rise to increasing effective disorder with increasing $\mathrm{T}$, and hence increasing $\rho(T)$ with T.) Note that the overall resistivity scale depends on the unknown impurity densities, but the qualitative trends in $\rho(T, n)$ arise from basic aspects of the underlying scattering mechanisms. 
We pointed out earlier [8] that the requirements for the observation of a large temperature-induced change in resistivity is the strong screening condition, $q_{T F} \gg$ $2 k_{F}$, where $q_{T F}$ and $k_{F}$ are the Thomas-Fermi screening wave vector and Fermi wave vector, respectively. Since $q_{T F} / k_{F} \propto g_{v}^{3 / 2}$ we expect the observed metallicity to be much stronger for $g_{v}=6$ than for $g_{v}=2$. The dashed lines in the Fig. 2 show the calculated resistivity for $n=4.5,4.9,5.36 \times 10^{11} \mathrm{~cm}^{-2}$ with $g_{v}=6$. We find, in the range of of $0-5 \mathrm{~K}$, roughly a factor of three change in $\rho(T)$ with $g_{v}=6$ whereas more like a $50 \%$ temperature induced change in $\rho(T)$ with $g_{v}=2$, which is consistent with the experimental observation. Thus, the transport measurement in $\mathrm{Si}(111)$-vacuum FET supports the Si(111) surface having a valley degeneracy 2 .

Finally, we briefly discuss the possible origin of the valley degeneracy puzzle in this system, i.e. why $g_{v}=2$ in the $\mathrm{Si}(111)$-vacuum FET system rather than the usual effective mass prediction of $g_{v}=6$. This is, in fact, an old problem [3] much discussed in the context of $\mathrm{Si}(111)-\mathrm{SiO}_{2}$ MOSFETs. It is generally accepted $[5,6]$ that the random strain associated with the Si surface lifts the valley degeneracy lowing two of the six valleys in energy. Only specially processed $\mathrm{Si}(111)-\mathrm{SiO}_{2}$ samples with rather low mobilities were ever found to have $g_{v}=6[6]$ with most $\mathrm{Si}(111)-\mathrm{SiO}_{2}$ MOSFETs having $g_{v}=2$. We believe the same kind of one electron physics to be operational in lifting the valley degeneracy from $g_{v}=6$ to $g_{v}=2$ in the $\mathrm{Si}(111)$-vacuum system as well although understanding the details of this valley degeneracy puzzle is beyond the scope of our current work where we are interested in the transport properties. We do, however, mention that this valley degeneracy lifting is unlikely to be a many-body exchange instability, which would occur even as a matter of principle at a much lower critical density $\left(\sim 10^{11}\right.$ $\mathrm{cm}^{-2}$ ) with a critical temperature of only $1 \mathrm{~K}$ or so. At the experimental density and temperature range of ref. [1], a many-body effect induced valley degeneracy lifting is extremely unlikely $[10,11]$

In conclusion, we calculate the the density and temperature dependent mobility and resistivity for the recently fabricated $\mathrm{Si}(111)$-vacuum field effect transistor by using a semiclassical Boltzmann theory including screened charged impurity scattering. We find reasonable agreement with the corresponding experimental transport properties for the (111) system indicating that the screened potential from random charged impurities is the main scattering source. In comparing our calculation with the existing experimental data $[1,2]$ we find that the calculated results with the valley degeneracy $g_{v}=2$ give better agreement with experiments than $g_{v}=6$.

This work is supported by LPS.
[1] K. Eng, R. N. McFarland, and B. E. Kane, Appl. Phys. Lett. 87, 052106 (2005); Physica E 34, 701 (2006).

[2] K. Eng, R. N. McFarland, and B. E. Kane, unpublished.

[3] T. Ando, A. B. Fowler, and F Stern, Rev. Mod. Phys. 54 437 (1982).

[4] B. E. Kane, L. N. Pfeiffer, K. W. West, and C. K. Harnett, Appl. Phys. Lett. 63, 2132 (1993); M. P. Lilly, J. L. Reno, J. A. Simmons, I. B. Spielman, J. P. Eisenstein, L. N. Pfeiffer, K. W. West, E. H. Hwang, and S. Das Sarma, Phys. Rev. B 90, 056806 (2003).

[5] D. C. Tsui and G. Kaminsky, Solid State Commun. 20, 93 (1976).

[6] D. C. Tsui and G. Kaminsky, Phys. Rev. Lett. 42, 595 (1979).

[7] T. Cole and B. D. McCombe, Phys. Rev. B 29, 3180 (1984).

[8] S. Das Sarma and E. H. Hwang, Solid State Comm. 135, 579 (2005); Phys. Rev. Lett. 83, 164 (1999); Phys. Rev. B 69, 195305 (2004); Phys. Rev. B 61, R7838 (2000).

[9] F. Stern, Phys. Rev. Lett. 44, 1469 (1980).

[10] G. Beni and T. M. Rice, Phys. Rev. B 20, 5390 (1979).

[11] S. Das Sarma and B. Vinter, Phys. Rev. B 28, 3639 (1983). 